\title{
High-speed interferometric FBG interrogator with dynamic and absolute wavelength measurement capability
}

\author{
Marcus Perry, Philip Orr, Pawel Niewczas, Members, IEEE, and Michael Johnston
}

\begin{abstract}
A passive, interferrometric wavelength demodulation technique has been extended to measure the absolute wavelengths of a multiplexed array of fiber Bragg grating sensors. The scheme retains its original strain resolution of $10 \mathrm{n} \varepsilon / \sqrt{\mathrm{Hz}}$. A proof-of-concept interrogation system was able to determine the absolute wavelength of Bragg peaks to within $20 \mathrm{pm}(17$ $\mu \varepsilon)$. Static and dynamic Bragg grating strains were accurately demodulated in both absolute and relative wavelength measurement modes. This demonstration indicates that interferometric techniques are able to provide absolute, static and dynamic measurements of strain within a single platform.
\end{abstract} tors

Index Terms - fiber Bragg gratings, interferometric, interroga-

\section{INTRODUCTION}

$\mathbf{F}$ IBER Bragg gratings (FBGs) offer reliable, wavelengthencoded measurements of strain-inducing parameters, that are immune to the interference and intensity fluctuations which plague many conventional electronic and optical transducers [1]. Their small, non-intrusive size, light weight and chemical inertness make them highly desirable for monitoring within civil, aerospace, energy and power engineering sectors [2].

One of the major strengths of FBGs is their ability to form quasi-distributed sensor networks. To take advantage of this, interrogators need to be able to measure and track the reflected peak wavelengths from a multiplexed array. Common methods involve actively scanning wavelength filters to allow the interrogator to map the spectrum, identify and then track peak locations [3]. However, interrogators based on passive interferometry are usually able to provide a far higher resolution, so it is of interest to develop these schemes further [4].

In previous work, we combined a passive, robust, interferometric wavelength-demodulation technique with fast optical pass-band switching to rapidly interrogate a multiplexed FBG array [5]. Static and dynamic strain resolutions as high as 1 $\mathrm{n} \varepsilon$ and $10 \mathrm{n} \varepsilon / \sqrt{\mathrm{Hz}}$ were achieved respectively [6], but due to the periodic nature of interferometer phase, these schemes were only able to measure relative changes in the wavelength of each sensor after the system was initialised.

Manuscript received Apr 00, 2013; revised May 00, 2013. This work was supported by the Engineering and Physical Sciences Research Council.

M. Perry, P. Orr and P. Niewczas are with the Institute for Energy and Environment, University of Strathclyde, Glasgow, UK.(phone: +44(0) 141548 4841; e-mail: marcus.perry@ strath.ac.uk.

M. Johnston is with EDF Energy Nuclear Generation Ltd, East Kilbride, UK.
In this article, we demonstrate how optical path length tuning can extend this system's measurement capabilities, and use it to retrieve the initial absolute wavelengths of a multiplexed array of fibre Bragg gratings. The static and dynamic strain measurement performance of a proof-of-concept laboratory scheme is scrutinised, to demonstrate that relative and absolute measurement capabilities are retained and extended. A comparison with simulated systems allows us to suggest future improvements to the scheme, which may help break the long-standing trade-off between multiplexing capability and wavelength measurement quality.

\section{SYSTEM DESIGN AND THEORY}

The interrogation scheme is shown in Figure 1. Bragg reflections from an FBG sensor array $\left(\lambda_{1}, \ldots, \lambda_{N}\right)$ are spectrally filtered and separated by an arrayed waveguide grating with uniform pass bands. An optical path switch sequentially passes the isolated Bragg peaks to an unbalanced Mach-Zehnder interferometer (MZI). The phase difference at the output of the MZI is wavelength dependent:

$$
\phi=\frac{2 \pi n D}{\lambda}
$$

where the optical path difference (OPD), $n D$, comprises the refractive index, $n(\lambda)$, and physical length difference between the arms, $D$. The interferometer's interference fringes modulate each Bragg reflection, producing a unique output intensity pattern over the interval $\phi=[0,2 \pi]$. For an MZI with three outputs, the intensities, once integrated by photodetectors, yield the following voltages $(m=1,2,3)$ :

$$
\overline{V_{m}}=\frac{V_{m}}{C_{1}}=\frac{C_{m}}{C_{1}}+\frac{D_{m}}{C_{1}} \cos \left(\phi+\theta_{m}\right)
$$

Here $\theta_{m}=2 \pi(m-1) / 3$, while $C_{m}$ and $D_{m}$ are parameters which quantify the mean and alternating components of the fringes for each channel. These simulatenous equations allow changes in phase to be expressed independently of confounding variables, such as fluctuations in optical source power and attenuation [7]:

$$
\tan (\phi)=\frac{\left(\mu_{2}-\mu_{3}\right) \bar{V}_{1}+\left(\mu_{3}-\mu_{1}\right) \bar{V}_{2}+\left(\mu_{1}-\mu_{2}\right) \bar{V}_{3}}{\left(\gamma_{2}-\gamma_{3}\right) \bar{V}_{1}+\left(\gamma_{3}-\gamma_{1}\right) \bar{V}_{2}+\left(\gamma_{1}-\gamma_{2}\right) \bar{V}_{3}}
$$

The normalisation parameters, $\mu_{m}=\frac{D_{m}}{C_{m}} \cos \left(\theta_{m}\right)$ and $\gamma_{m}=$ $\frac{D_{m}}{C_{m}} \sin \left(\theta_{m}\right)$, depend on the fringe depth and mean output intensity of the MZI, and the spectral shape of each FBG. 


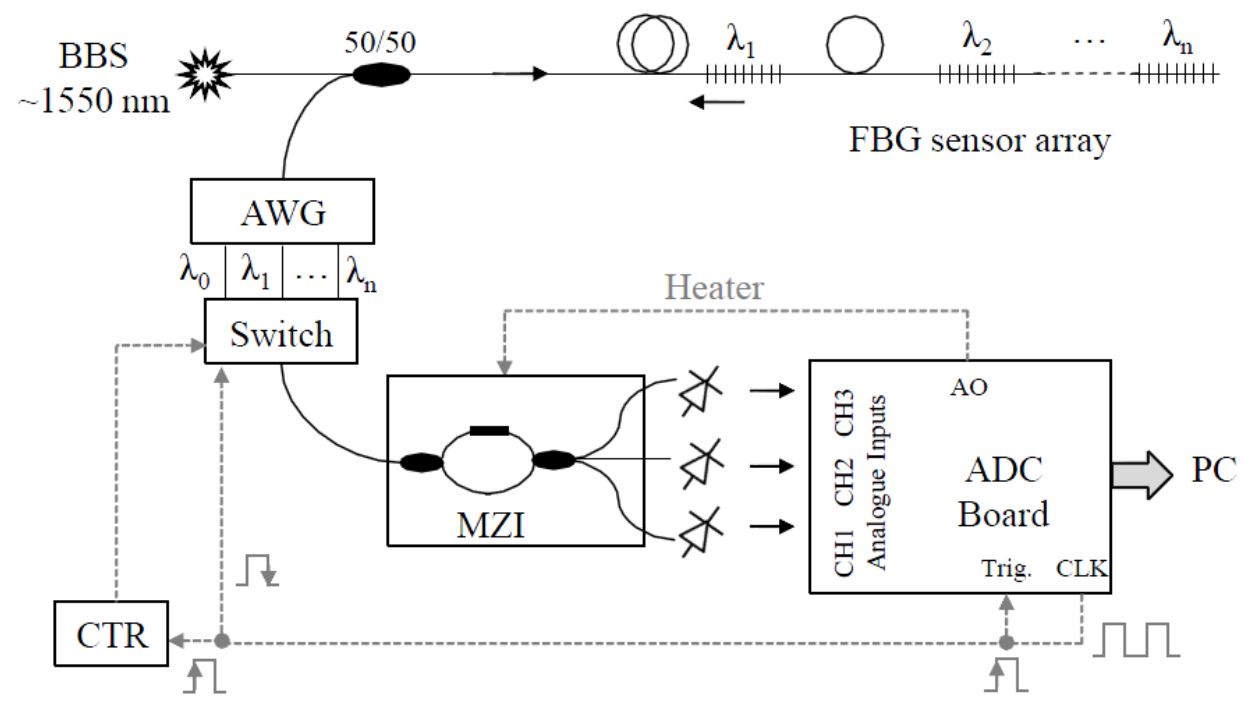

Fig. 1. Multiplexed FBG interrogator, with optical and electrical connections shown in black and gray respectively. The FBG sensor array is illuminated by a broadband source (BBS). Bragg reflections are sequentially passed to the Mach-Zehnder interferometer (MZI) by using an arrayed waveguide grating (AWG) and optical path switch. The ADC board supplies the signals required for optical switching and thermal tuning of the MZI.

These parameters also capture the fact that the values of $\theta_{m}$ may not be perfectly separated by $120^{\circ}$ in a real system.

\section{A. Phase calibration}

In previous work, normalisation parameters, $\mu_{m}$ and $\gamma_{m}$, were calculated by thermally or mechanically tuning a reference Bragg peak across the interferometer's fringes. During this calibration procedure, the amplitudes and dc offsets of the voltage responses were used in conjunction with a technique developed by Hereth and Schliep to evaluate $\mu_{m}$ and $\gamma_{m}$ [8]. The calculated parameters for this FBG were then applied to all others in the array.

In this paper, the method is reversed - instead, the fringes are scanned over all Bragg peaks simultaneously by tuning the interferometer's path difference. This not only facilitates the absolute wavelength calibration discussed later, but also provides normalisation parameters which are unique to each FBG. Thermally tuning the path imbalance by heating a length section, $L$, of one arm of the interferometer results in a linear phase shift with temperature, $T$ :

$$
\frac{d \phi}{d T}=\frac{2 \pi}{\lambda}\left(D \frac{d n}{d T}+n L \alpha\right)
$$

where $\frac{d n}{d T}$ and $\alpha$ are thermo-optic and thermal expansion coefficients respectively.

As thermal tuning alters the fiber's refractive index (and hence absorption losses in the MZI), $\gamma_{m}$ and $\mu_{m}$ are also temperature dependent. As shown in Figure 2, this dependence is characterised at discrete temperatures by detecting the peaks and troughs in the voltage responses during thermal tuning. The amplitudes and offsets of the three interferograms can then be estimated continuously by interpolation, providing $\gamma_{m}(T)$ and $\mu_{m}(T)$.

The temperature-dependent normalisation parameters are finally applied, using equation (3), to the same voltage history from which they were generated. This determines the phase shift for each FBG during thermal tuning. Using the same data for both calibration and phase interpretation improves measurement accuracy and also reduces the number of steps in the method.

\section{B. Absolute wavelength}

It is clear from equation (2) that channel voltages periodically repeat as the Bragg peak shifts over the free spectral range of the interferometer. Multiples of $2 \pi$ phase shifts are tracked and unwrapped for each FBG during system operation, but the number of fringes initially separating two FBGs is not generally known. As such, interferometric schemes like this have been historically limited to tracking only the relative wavelength shifts between sensor and reference FBGs.

However, in the latest developments reported here, the absolute wavelength of each sensor can be measured when the system is initialised [9]. This is possible because the thermal tuning procedure, described above, produces large and accurately monitored phase measurements for each FBG. The wavelength of an unknown sensor at $\lambda_{1}$ can therefore be calculated from a known reference, situated at $\lambda_{r}$, using the ratio of the phase shifts:

$$
\frac{d \phi_{r}}{d T} \cdot \frac{d T}{d \phi_{1}}=\frac{d \phi_{r}}{d \phi_{1}}=\frac{\lambda_{1}}{\lambda_{r}}\left[\frac{D \frac{d n\left(\lambda_{r}\right)}{d T}+n\left(\lambda_{r}\right) L \alpha}{D \frac{d n\left(\lambda_{1}\right)}{d T}+n\left(\lambda_{1}\right) L \alpha}\right]
$$

In cases where, $D \frac{d n}{d T} \ll n L \alpha$, this relationship can be simplified to:

$$
\phi_{r}=\left(\frac{\lambda_{1}}{\lambda_{r}} \cdot \frac{n\left(\lambda_{r}\right)}{n\left(\lambda_{1}\right)}\right) \phi_{1}
$$

The relative refractive indices are calculated using the Sellmeier equation for fused silica: 


$$
n^{2}(\lambda) \approx 1+\frac{0.7 \lambda^{2}}{\lambda^{2}-4680}+\frac{0.4 \lambda^{2}}{\lambda^{2}-13500}+\frac{0.9 \lambda^{2}}{\lambda^{2}-10^{7}}
$$

and so the slope, $S$, of $\phi_{r}$ against $\phi_{1}$ can be corrected to find $\lambda_{1}$ without any knowledge of temperature. To re-iterate, the phase calibration and absolute wavelength procedures are both performed from the same voltage history during one thermal tuning operation, providing both the wavelength and phase response of each FBG sensor in a single step.

\section{Simulation}

A simulation of the system in Figure 1 was used to demonstrate the theoretical limit of precision of the absolute wavelength calculation. Phase demodulation of both simulated sensor $\left(\lambda_{1}=1540 \mathrm{~nm}\right)$ and reference $\left(\lambda_{r}=1560 \mathrm{~nm}\right)$ Bragg peaks was performed using Todd's passive algorithm [7]. A phase noise floor of $250 \mu \mathrm{rad} / \sqrt{\mathrm{Hz}}$ was introduced, as this corresponds with the noise floor of the laboratory scheme. To simulate thermal tuning, the initial OPD of $4.6 \mathrm{~mm}$ was detuned by $1 \%$ at a rate of $1 \mu \mathrm{m} / \mathrm{s}$ and phases were sampled at a rate of $500 \mathrm{~Hz}$.

A histogram of the calculated sensor wavelength over 10,000 iterations is shown in Figure 3. The typical fractional error in the slope of $\phi_{1}$ against $\phi_{r}$ was $\Delta S=1.2 \times 10^{-6}$. This lead to a standard error in the wavelength of $1.8 \mathrm{pm}$, equivalent to $0.14{ }^{\circ} \mathrm{C}$ or $1.5 \mu \varepsilon$.

\section{LABORATORY DEMONSTRATION}

To better characterise the performance of the technique, a proof-of-concept system, based on Figure 1, was constructed with a limited number of FBGs. An athermal grating (temperature sensitivity $<0.5 \mathrm{pm} /{ }^{\circ} \mathrm{C}$ ) at $\lambda_{r}=1560 \mathrm{~nm}$ was used as a stable reference. Its phase response was initially compared to another athermal grating at $\lambda_{1}=1540 \mathrm{~nm}$, as this allowed the absolute wavelength calibration to be verified without the extraneous influence of Bragg wavelength uncertainty.

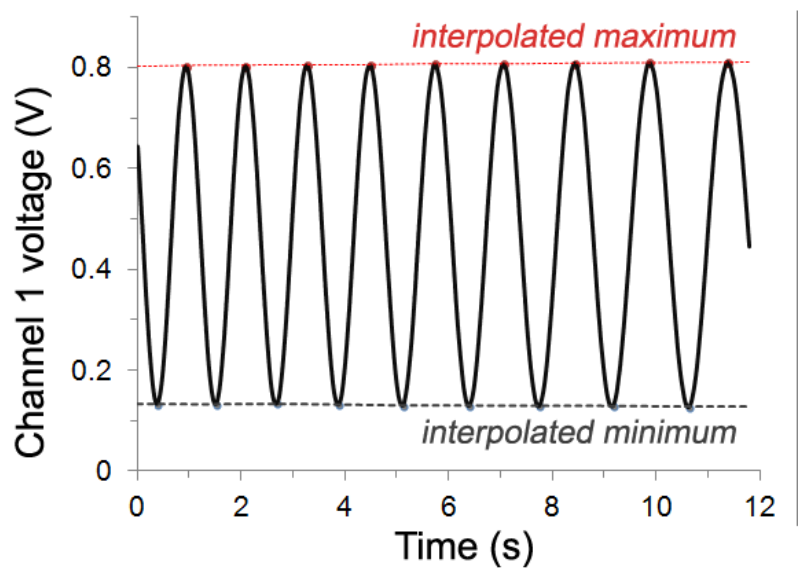

Fig. 2. The photodetector voltage obtained for one MZI channel during thermal tuning. Interpolation between peak-detected maximum and minimum values allows the ac and dc components of the waveform to be estimated continuously as a function of time. This allows normalisation parameters to be constructed as functions of temperature.

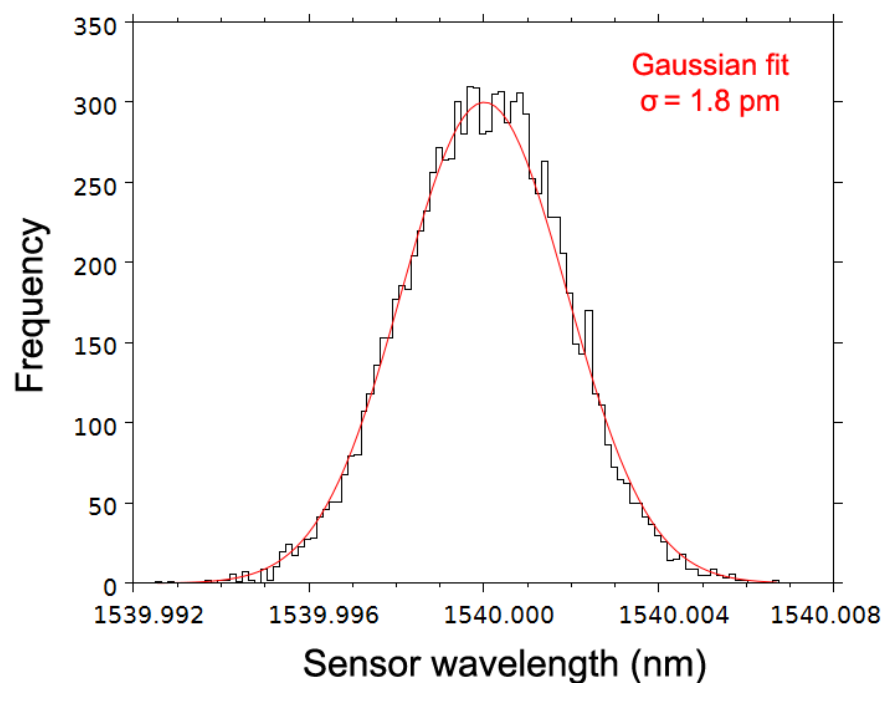

Fig. 3. Histogram of the calculated absolute wavelength of an FBG at $1540 \mathrm{~nm}$ after 10,000 simulated iterations. The standard deviation, $\sigma$, of the Gaussian fit is $1.8 \mathrm{pm}$. Bragg peak spectral widths were $0.1 \mathrm{~nm}$. FBG reflectivity and MZI fringe depth were both set to $80 \%$.

The interferometer (OPD $4.6 \mathrm{~mm})$ was an all-fiber construction, fabricated by splicing the pigtails of $1 \times 2$ and $3 \times 3$ couplers. To isolate the MZI from vibration and to hinder heat transfer between the arms during thermal tuning, the device was bonded to a glass slab by epoxy. Tuning was achieved by resistive heating an $L=4 \mathrm{~cm}$ section of one fiber arm. Heat conduction was facilitated by thermal grease. The ADC unit sampled at $4 \mathrm{kHz}$, and each set of four data points were averaged. After switching between the two FBGs, this provided a measurement rate of $500 \mathrm{~Hz}$ per sensor.

\section{A. Initial calibration}

The procedures for calibration and absolute wavelength determination are summarised in Figure 4. Normalisation parameters and phase shifts for both FBGs were calculated continuously over 20-30 seconds of thermal tuning. The empirical relationship between the phase shift of the reference FBG and the resistive heater's temperature is shown in Figure 5. The slope of this fit describes equation (4), and substituting $\frac{d n}{d T}=8.5 \times 10^{-6}$ for silica glass suggests that thermal expansion in the arm was around $\alpha=9 \times 10^{-6}$. This is a sensible value, mid-way between the thermal expansion of silica and epoxy, and so provides verification that phase shifts were measured accurately.

\section{B. Absolute wavelength resolution}

The phase relationship between the reference and unknown FBGs during thermal tuning is shown in Figure 6(a). The uncorrected slope of the linear fit is $S=1.01316 \pm 10^{-5}$. As shown in Figure 6(b), the refractive index correction factor is found numerically to yield, in this case, $\lambda_{1}=1539.989$ $\pm 0.003 \mathrm{~nm}$.

The quoted $3 \mathrm{pm}$ wavelength error is derived from phase noise and is thus comparable to the precision found in the simulation. To experimentally verify this wavelength uncertainty, 


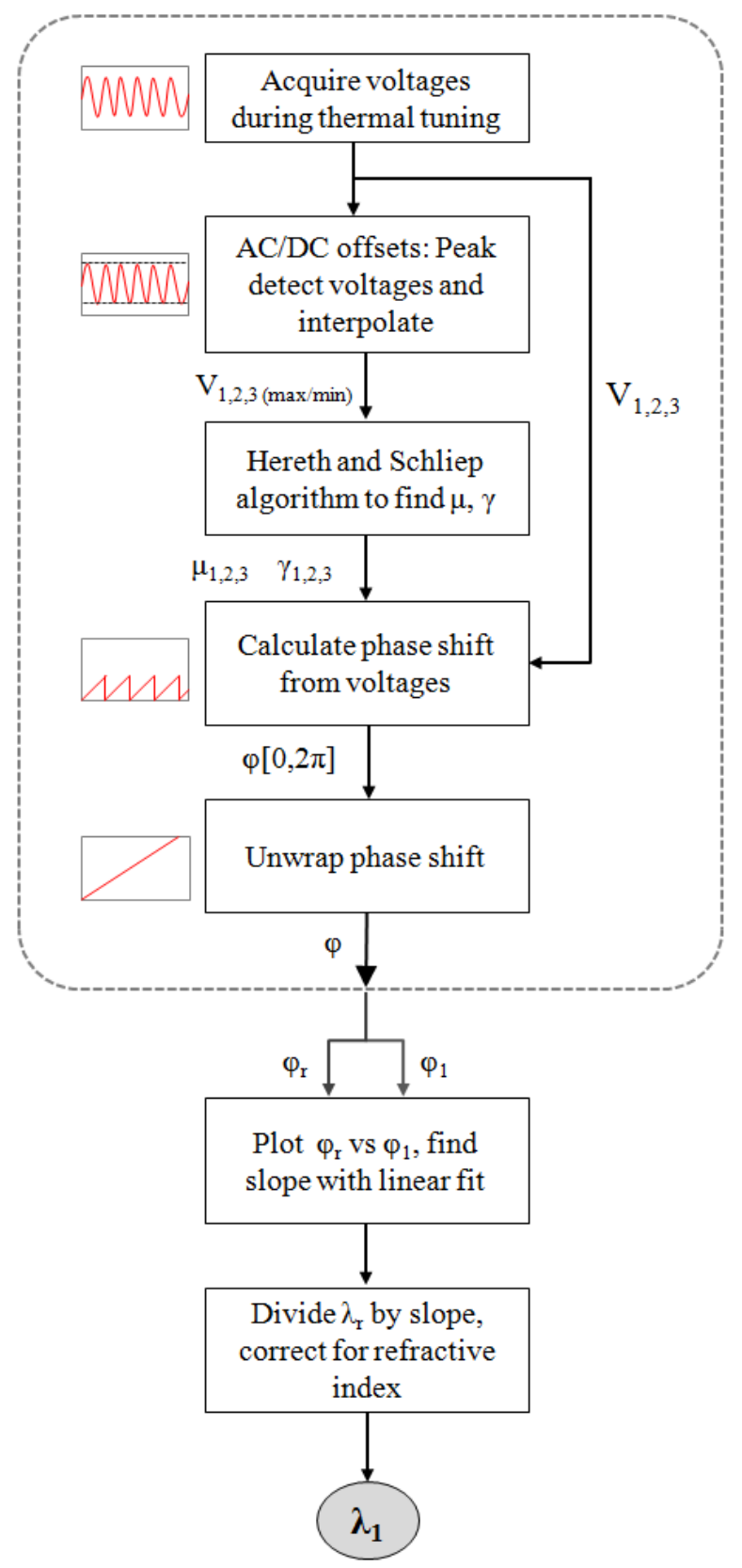

Fig. 4. Flowchart of the steps in the calculation of absolute wavelength. Channel voltages were translated into phase shifts for the reference and unknown FBGs during thermal tuning. The relative phase shifts are then used to calculate the wavelength of the unknown FBG.

tuning and calibration was repeated 30 times. The resulting histogram, shown in Figure 7, reveals that the calculated sensor wavelength was accurate to within $20 \mathrm{pm}$, with a standard error of $15 \mathrm{pm}$ (equivalent to $1.2{ }^{\circ} \mathrm{C}$ or $12.5 \mu \varepsilon$ ). Precision and accuracy are lower than expected, due to the influence of uncertainty in the calibration parameters. Initially, these parameters were assumed to be perfectly known, but realistically, uncertainty in $\mu_{m}$ and $\gamma_{m}$ can have a profound impact on the phase calculation.

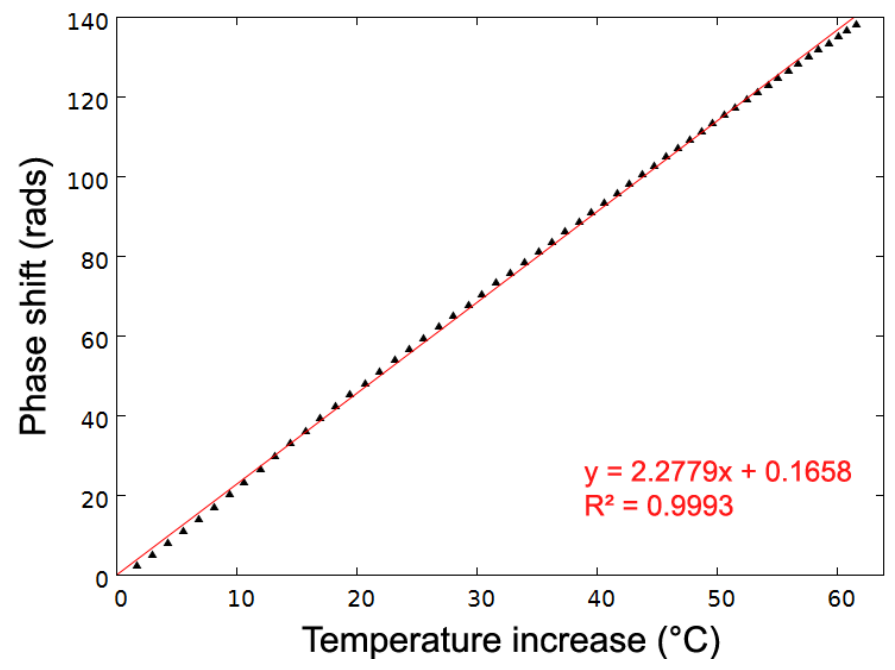

Fig. 5. Resistive heater temperatures were measured using an FBG thermometer. As the temperature of one arm of the MZI was increased, phase was found to shift linearly, confirming equation (4). The R-squared value for the fit is 0.9993 .
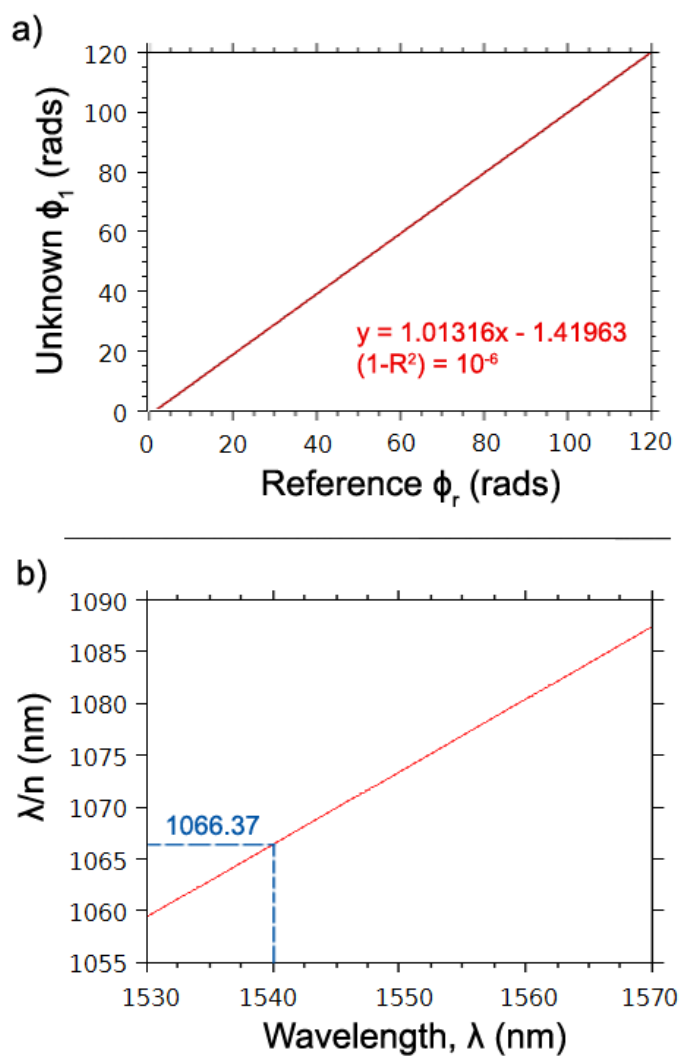

Fig. 6. a) Experimental data of the phase relationship between the reference and unknown FBGs during thermal tuning. The slope of the linear fit is $S=$ $1.01316 \pm\left(7 \times 10^{-6}\right)$. The R-squared value of the linear fit is approximately 1. b) The correction factor for the slope is found by solving equation (6) numerically. A plot of $\lambda$ against $\lambda / n(\lambda)$ is used. When $\lambda / n=1560 /[S \times$ $n(1560)]$ the corrected absolute wavelength is found to be $\lambda_{1}=1539.989$ $\mathrm{nm}$. 


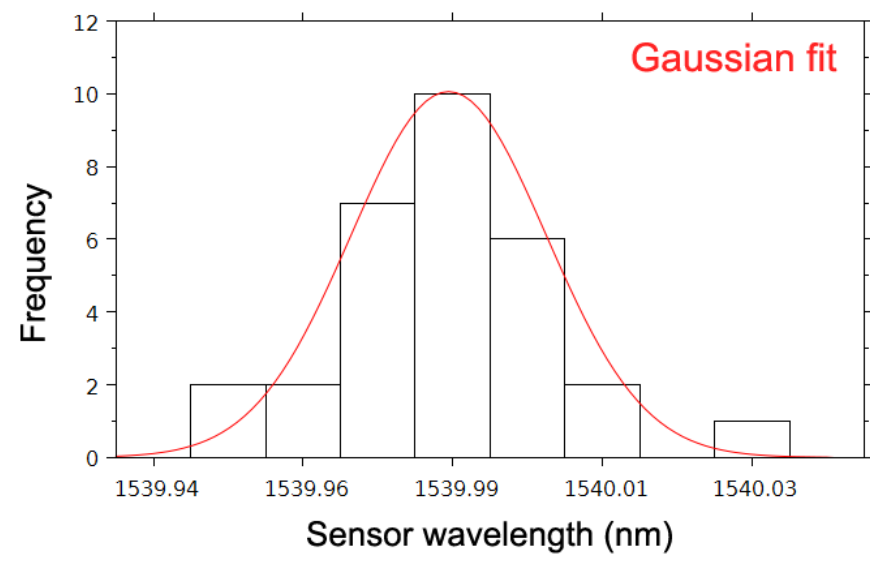

Fig. 7. Histogram and Gaussian fit of the experimental absolute wavelength calculation of an athermal FBG at $1540 \mathrm{~nm}$ after 30 iterations. The mean and standard deviation of the data was 1539.98 and $15 \mathrm{pm}$. A t-test suggests $99 \%$ confidence that the true sample mean lies within $\pm 10 \mathrm{pm}$ of the calculated value.

\section{Dynamic strain measurements}

A key feature of the proposed scheme is its ability to provide both absolute and dynamic measurements of FBG wavelengths. To analyse the system's dynamic measurement performance, a sensor FBG at $\lambda_{2}=1550 \mathrm{~nm}$ was mounted on a cantilever using epoxy. Because thermal tuning is timeconsuming, it is not suitable for real-time monitoring of absolute wavelengths during dynamic loading of an FBG. However, once the absolute wavelength is initially calculated once, subsequent high-speed measurements of relative phase shifts can be used to infer the new wavelength state.

To demonstrate this concept, the cantilever tip was deflected to induce an initial wavelength offset in the FBG. The interrogation system was switched on and the thermal tuning procedure was used to locate the sensor's wavelength. Once the MZI's temperature had restabilised, the cantilever was released. As shown in Figure 8, by combining absolute and relative strain measurements, the system was able to identify both the initial $2.5 \mathrm{~m} \varepsilon$ offset and the subsequent decaying oscillations about zero. A Fourier transform of these oscillations also reveals a noise floor of around $10 \mathrm{n} \varepsilon / \sqrt{\mathrm{Hz}}$.

\section{Static strain measurements}

The system's ability to monitor static strain was also evaluated. The cantilever was deflected and then held stationary in five stages, placing the attached FBG under strains ranging from 0.6 to $2.6 \mathrm{~m} \varepsilon$. The wavelength of the FBG could be determined either by repeatedly calibrating to find the absolute wavelength after each step in cantilever deflection, or the shifts measured during deflection could be used to infer the new wavelength from a single initial value. The wavelengths obtained using both of these methods were compared to 'actual values', verified using a commercial Fabry-perot filter based interrogation unit.

Figure 9 shows that both absolute and relative modes were able to accurately find the 'true' wavelength of the FBG as it changed. The stability of relative wavelength measurements is currently limited compared to previously reported systems because this prototype MZI is not yet fully thermally isolated or stabilised. Note that obtaining absolute values of strain by repeated thermal tuning is an active, slow method of demodulation, only used here to demonstrate the accuracy of the absolute wavelength technique. In a real application, absolute wavelength calibration would only be performed once initially. Relative wavelength shifts from this initial value would then be measured, as the interrogation rate and accuracy of the relative mode is superior.

\section{Discussion}

There are several contributions to error which may have prevented the prototype system from achieving the simulated measurement performance. For example, uncertainty or inaccuracy in the reference wavelength has an impact both on the phase and refractive index calculations. The athermal reference FBGs were highly stable, but superior accuracy may be achieved by stabilising with the absorption lines from gas cells, provided these can be guided by the same optical components [10], [11].

Matching the spectral width of the FBGs to the free spectral range of the MZI is also crucial. In this work, the measurement resolution of athermal FBGs was better not only because of their temperature stability, but also because their spectral width $(0.1 \mathrm{~nm})$ was lower than that of the cantilever-bound FBG $(0.3 \mathrm{~nm})$. Spectrally narrow reflections allow for a more pronounced modulation of channel voltages, improving the signal to noise ratio. Using, for example, line emission references with fiber laser sensors would improve system performance in both absolute and relative modes.

In this prototype system, the lack of adequate thermal damping between the MZI and the environment made static measurements challenging. In previous systems, a dielectric gel was used to thermally insulate the interferometer [6]

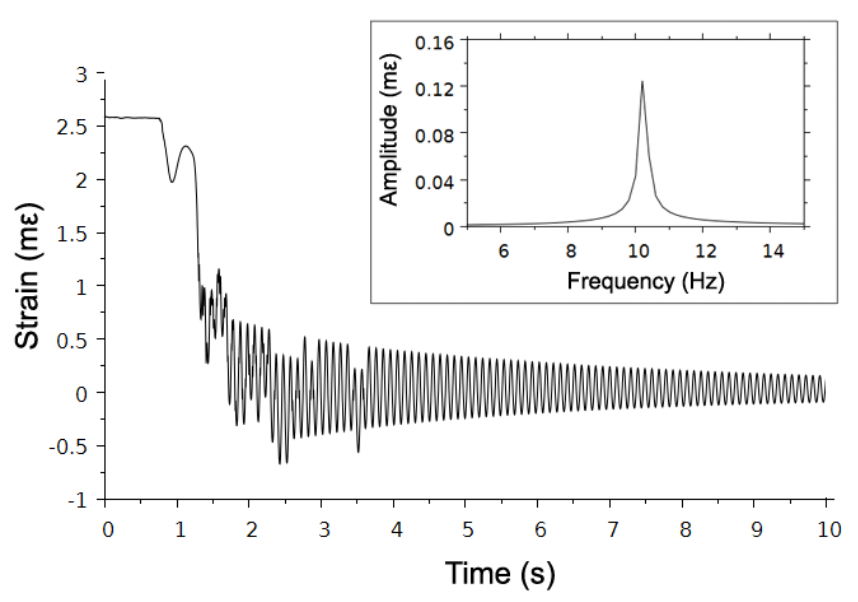

Fig. 8. Strain measurements from a cantilever-mounted FBG. Before interrogation began, the cantilever was initially held to induce a strain of approximately $2.5 \mathrm{~m} \varepsilon$ in the FBG. The system was able to interrogate the initial absolute strain and then monitor the subsequent relative changes when the cantilever was released. Shown inset, the Fourier transform of the data between 5 and $10 \mathrm{~s}$, reveals that the cantilever oscillates at its natural frequency of $10.2 \mathrm{~Hz}$. The noise floor was $10 \mathrm{n} \varepsilon / \sqrt{\mathrm{Hz}}$. 
and in practical implementations, standard telecommunication component packaging including active temperature control can provide thermal stability. For dynamic measurements, thermal drift is less of a concern as unwanted low-frequency components can be filtered out.

The success of the relatively simple prototype, demonstrated here, encourages further study. In the next evolution of the system, we propose that the MZI is miniaturised by incorporating it onto a silicon photonics platform [12]. This should improve stabilisation and reduce the time taken to thermally tune the path difference to within tens of milliseconds [13].

Such a platform introduces new challenges, however. In the current system, the high thermal expansion of the epoxy and the low thermo-optic coefficient of silica glass allow refractive index correction factors to be calculated with the simplified equation (6). Arguably, this simplification may have been partly responsible for some loss of accuracy. Improvements could be made by using the more complicated correction factor in equation (5), but this requires detailed knowledge of parameters such as the heated length of waveguide and the wavelength-dependence of the thermo-optic coefficient. These parameters are not only difficult to measure, but may also themselves harbour some temperature dependence. It may therefore be desirable to design the MZI such that simplifications can be made.

When dealing with miniaturised silicon photonics, the high thermo-optic coefficient leads to a situation which is the reverse of the present one, where $D \frac{d n}{d T} \gg n L \alpha$. It may eventually be found that simplifications cannot be made at all, but the parameters can at least be more accurately known and controlled through careful design.

The ratio of the thermal tuning and sampling speed of the system defines parameters such as uncertainty in the slope, $\Delta S$. The higher thermal tuning rate of a miniaturised platform thus requires similar improvements to the sampling frequency. In the prototype system, the relatively low $500 \mathrm{~Hz}$ sampling rate only produced slope errors corresponding to a $3 \mathrm{pm}$ wavelength uncertainty. A much larger error arose from imperfect knowledge of the calibration parameters, but this was also the result of phase noise. Thermal tuning speeds may therefore have to be deliberately limited to allow for accurate and precise deduction of $\mu_{m}(T)$ and $\gamma_{m}(T)$. Faster tuning may allow several absolute wavelength calibrations to be rapidly made in series, however, especially if calibration could be performed also during cooling. This would produce a result with higher confidence, from a statistical viewpoint. However, because absolute wavelength calibration requires only a single tuning event, there may also be some benefit to modelling $\mu_{m}$ and $\gamma_{m}$ to gain a better understanding of how these parameters evolve with temperature, .

\section{CONCLUSION}

A fiber Bragg grating interrogation scheme based on a triple-output interferometer has been extended to provide measurements of absolute sensor wavelengths, in addition to its previous ability to measure relative wavelength shifts. By thermally tuning the interferometer once, the phase responses

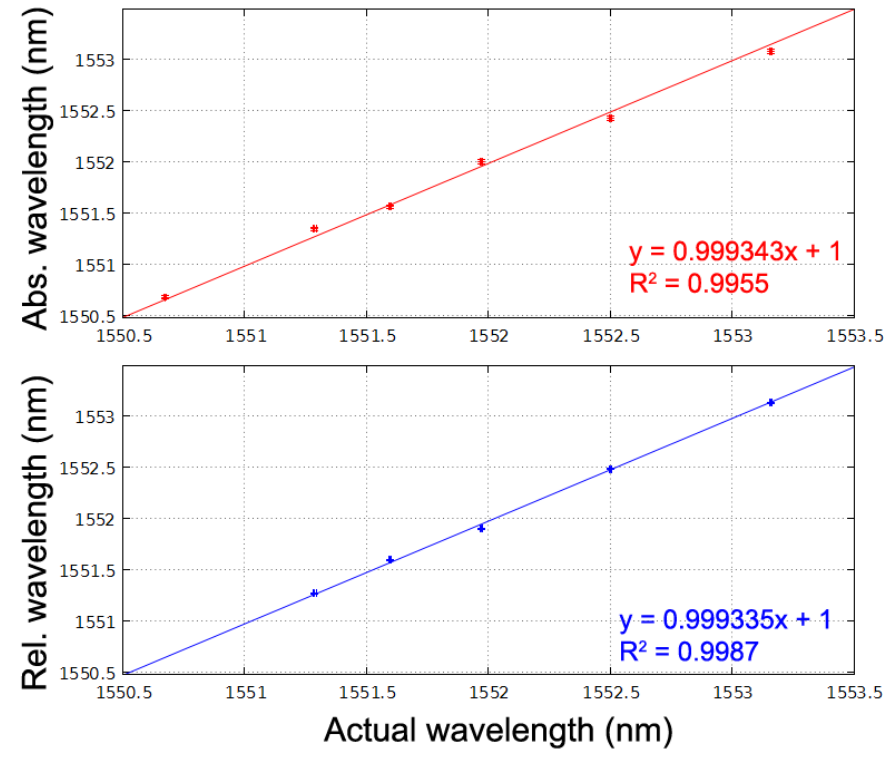

Fig. 9. The 'actual' wavelength of a cantilever-mounted FBG, measured using a commercial interrogator (resolution $5 \mathrm{pm}$ ), compared to the wavelengths measured by the system. Absolute wavelengths were monitored by repeatedly using the thermal tuning technique while the cantilever was stationary. Relative wavelengths were inferred by summing the phase shifts while the cantilever was undergoing deflection and then adding these to the initial value. The gradients of both lines should ideally equal unity, and this is the case to at least 3 significant figures, showing that both modes are able to record wavelength accurately.

of each sensor in the array were simultaneously characterised and compared. Because phase shifts are wavelength dependent, the phase shifts of unknown sensors were compared to those of a known reference to provide an absolute measure of the wavelength of each sensor.

A prototype system revealed that the absolute wavelengths calculated were both accurate and precise to within $20 \mathrm{pm}$ (equivalent to $1.6{ }^{\circ} \mathrm{C}$ or $17 \mu \varepsilon$ ). Both accuracy and precision were limited by errors in the characterisation of the interferometer's output response and its dependence on temperature during thermal tuning. The system was able to accurately resolve static and dynamic Bragg grating strains in both absolute and relative wavelength measurement modes.

The ability of the interrogator to determine absolute wavelength, while retaining the speed and resolution offered by interferometry, will contribute to making instrumentation by FBG sensors a far more attractive prospect to many industries. Additionally, the passive, solid-state nature of the interrogator lends itself to potential fabrication in planar optics formats, such as silicon photonics, which could in future lead to physically small, cost-effective and mass producible interrogation systems.

\section{REFERENCES}

[1] A. Kersey, M. Davis, H. Patrick, M. LeBlanc, K. Koo, C. Askins, M. Putnam, and E. Friebele, "Fiber grating sensors," Lightwave Technology, Journal of, vol. 15, pp. 1442-1463, Aug. 1997.

[2] A. Mendez, "Fiber bragg grating sensors: a market overview," in Third European Workshop on Optical Fibre Sensors, pp. 661905-661905, International Society for Optics and Photonics, 2007. 
[3] Y. Zhao and Y. Liao, "Discrimination methods and demodulation techniques for fiber bragg grating sensors," Optics and Lasers in Engineering, vol. 41, no. 1, pp. 1-18, 2004.

[4] B. Lee, "Review of the present status of optical fiber sensors," Optical Fiber Technology, vol. 9, no. 2, pp. 57-79, 2003.

[5] P. Orr and P. Niewczas, "High-speed, solid state, interferometric interrogator and multiplexer for fibre bragg grating sensors," J. Lightwave Techn, no. 99, pp. $1-1,2011$.

[6] M. Perry, P. Orr, P. Niewczas, and M. Johnston, "Nanoscale resolution interrogation scheme for simultaneous static and dynamic fiber bragg grating strain sensing," Journal of Lightwave Technology, vol. 30, no. 20, pp. 3252-3258, 2012.

[7] M. Todd, G. Johnson, and C. Chang, "Passive, light intensityindependent interferometric method for fibre Bragg grating interrogation," Elec. Lett., vol. 35, no. 22, pp. 784-786, 1999.

[8] F. Schliep and R. Hereth, "Phase sensitive measurement technique for singlemode fibre directional couplers," Electronics Letters, vol. 28, no. 16, pp. 1538-1540, 1992.

[9] P. Niewczas and P. Orr, "Optical fibre sensor interrogation system," Jan. 4 2013. WO Patent 2,013,001,268.

[10] W. Ecke, K. Schröder, A. Andreev, and R. Willsch, "Thermally stable optical fibre bragg grating wavelength reference," Optics Communications, vol. 284, no. 6, pp. 1557-1560, 2011.

[11] M. A. Rowe, W. C. Swann, and S. L. Gilbert, "Multiple-wavelength reference based on interleaved, sampled fiber bragg gratings and molecular absorption," Applied optics, vol. 43, no. 17, pp. 3530-3534, 2004.

[12] B. Jalali and S. Fathpour, "Silicon photonics," Lightwave Technology, Journal of, vol. 24, no. 12, pp. 4600-4615, 2006.

[13] C. Holmes, D. Kundys, J. Gates, C. Gawith, and P. Smith, "150 ghz of thermo-optic tuning in direct uv written silica-on-silicon planar bragg grating," Electronics letters, vol. 45, no. 18, pp. 954-956, 2009.

Marcus Perry received an MSci degree in Physics from the University of Bristol, UK in 2009. He is currently working with EDF Energy towards an Engineering Doctorate in optical fiber sensors for use in nuclear environments at the Institute for Energy and Environment, University of Strathclyde. His research focuses on the use of fiber Bragg grating strain sensors for monitoring prestress loss in fission reactor prestressed concrete pressure vessels.

Philip Orr received the BEng and $\mathrm{PhD}$ degrees in electronic and electrica engineering in 2007 and 2011 respectively from the University of Strathclyde, where he is presently a research associate with the Institute for Energy and Environment. His core research area is the fundamental design of fiber sensors and interrogators for application within power and energy systems. In particular, he is concerned with intrinsic fiber sensing, multiplexing topologies, electromagnetic field measurement, nuclear diagnostics, and power system protection.

Pawel Niewczas received his $\mathrm{PhD}$ in the area of optical current sensors from the University of Strathclyde in 2000. He is currently a Senior Lecturer in the Department of Electronic and Electrical Engineering, University of Strathclyde, and is leading the Advanced Sensors Team within the Institute for Energy and Environment in the same department. His main interests centre on the advancement of optical sensing methods in such areas as power system metering and protection; gas turbine monitoring; downhole pressure, temperature, voltage and current measurement and in nuclear fission and fusion environments. He has published over 60 technical papers in this area.
Michael Johnston received a BA degree in Natural Sciences in 1989 and a PhD degree in Materials Science in 1994, both from the University of Cambridge. He currently works in the Civil Design Group, Plant Engineering, of EDF Energy Nuclear Generation Ltd. His job roles include managing the civil engineering part of the EDF Energy Nuclear Generation strategic research programme, which includes projects to develop enhanced methods of monitoring nuclear safety-related structures. 